Canadian

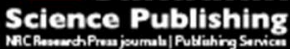

Applied Physiology, Nutrition, and Metabolism Physiologie appliquée, nutrition et métabolisme

\title{
Influence of exercise training with resveratrol supplementation on skeletal muscle mitochondrial capacity
}

\begin{tabular}{|r|l|}
\hline Journal: & Applied Physiology, Nutrition, and Metabolism \\
\hline Manuscript ID & apnm-2015-0370.R1 \\
\hline Manuscript Type: & Article \\
\hline Date Submitted by the Author: & 27-Aug-2015 \\
\hline Complete List of Authors: & $\begin{array}{l}\text { Polley, Kristine; University of Georgia, Kinesiology } \\
\text { Jenkins, Nathan; University of Georgia, Kinesiology } \\
\text { O'Connor, Patrick; University of Georgia; University of Georgia, Kinesiology } \\
\text { McCully, Kevin; University of Georgia, Kinesiology; The University of } \\
\text { Georgia }\end{array}$ \\
\hline Keyword: & $\begin{array}{l}\text { ergogenic aids < nutrition, muscle physiology < muscle physiology, muscle } \\
\text { metabolism < muscle metabolism, muscle function < muscle function, } \\
\text { muscle adaptation < muscle adaptation }\end{array}$ \\
\hline
\end{tabular}



mitochondrial capacity

4 Kristine R. Polley, Nathan Jenkins, Patrick O’Connor, Kevin McCully

6 Correspondence: Kevin McCully, 330 River Road, University of Georgia, Athens, 30602.

7 Phone: 706-542-1129, fax: 706-3148,mccully@uga.edu

9 Department of Kinesiology, 330 River Road, University of Georgia, Athens, GA, 30602, USA

10 (KP, NJ, PO, KM) kpolley@uga.edu, jenkinsn@uga.edu, poconnor@uga.edu, mccully@uga.edu 


\section{$\underline{\text { Abstract }}$}

14 Background: Physical inactivity reduces, and exercise training increases, mitochondrial

15 capacity. In rodents, exercise training effects can be augmented by large doses of resveratrol

16 supplementation but whether this can occur in humans with a smaller dose is unclear.

17 Objective: This study sought to determine the effects of resveratrol supplementation in

18 combination with exercise training on skeletal muscle mitochondrial capacity.

19 Design: Sixteen healthy young adults were randomly assigned in a double blind fashion to

20 consume either placebo or $500 \mathrm{mg}$ of resveratrol plus $10 \mathrm{mg}$ of piperine, a bioenhancer to

21 increase bioavailibilty and bioefficacy of resveratrol. Participants ingested the pills daily for 4

22 weeks and completed 3 sessions per week of submaximal endurance training of the wrist flexor

23 muscles of the non-dominant arm. The contralateral arm served as an untrained control.

24 Skeletal muscle mitochondrial capacity was measured using near-infrared spectroscopy (NIRS).

25 Results: Changes in mitochondrial capacity from baseline to post testing indicated significant

26 differences between the resveratrol+piperine trained arm and the placebo trained arm $(p=0.02)$,

27 with the resveratrol+piperine group increasing about $40 \%$ from baseline $(\Delta \mathrm{k}=0.58)$, while the

28 placebo group increased about $10 \%$ from baseline $(\Delta \mathrm{k}=0.13)$. Neither the placebo nor the

29 resveratrol+piperine group exhibited changes in mitochondrial capacity in the untrained arm.

30 Conclusions: Low intensity exercise training can increase forearm skeletal muscle mitochondrial

31 capacity when combined with resveratrol and piperine supplementation.

32 Key words: resveratrol, skeletal muscle, mitochondrial capacity, NIRS, exercise training,

33 submaximal endurance training 


\section{$\underline{\text { Introduction }}$}

Dietary supplements that target health and performance are a large global commercial market, with billions of dollars spent per year (Hardy 2000). One type of dietary supplement is

39 polyphenols, which are the most abundant antioxidants in the diet and have recently gained

40 attention due to their potential beneficial effects on metabolic health and aging (Scalbert et al.

41 2005; Schrauwen and Timmers 2014). A popular supplement known as resveratrol has been the

42 focus of much research due to its reported anti-inflammatory, anti-oxidant, and anti-tumorigenic 43 properties (Smoliga et al. 2011).

44 Resveratrol is a polyphenol antioxidant found in grapes, red wine, peanuts, cranberries, 45 and other plant species. Resveratrol has become of particular interest in the past decade in part

46 because of its potential ability to stimulate the expression of the SIRT1-AMPK-PGC1 $\alpha$ pathway

47 in skeletal muscle, leading to enhanced mitochondrial capacity (Lagouge et al. 2006; Timmers et

48 al. 2011; Ungvari et al. 2011; Menzies et al. 2013; Goh et al. 2014; Kulkarni and Canto 2014).

49 Exercise training also activates this pathway and results in enhanced mitochondrial capacity

50 (Winder et al. 2000; Jager et al. 2007; Hood 2009).

51 Several experiments in rodents have reported an augmented effect on skeletal muscle

52 mitochondrial capacity of exercise training when it was combined with resveratrol

53 supplementation (Murase et al. 2009; Dolinsky et al. 2012; Menzies et al. 2013). In humans,

54 there have been few studies assessing resveratrol's effect on mitochondrial capacity and the

55 research to date has produced contradictory findings. Some studies show upregulation of

56 mitochondria and the pathways that lead to increased mitochondrial capacity (Timmers et al.

57 2011; Goh et al. 2014), while others show minimal(Voduc et al. 2014) or attenuated training

58 adaptations (Gliemann et al. 2013; Olesen et al. 2014; Scribbans et al. 2014; Voduc et al. 2014) . 
59 These inconsistent findings in human studies could be due to multiple factors including the

60 health of the participants, variations in the muscles trained, the training stimulus, and the dose

61 and bioavailability of the resveratrol.

62 A concern in human studies is the low bioavailability of resveratrol (Amri et al. 2012).

63 Maximum plasma resveratrol concentrations $\left(\mathrm{C}_{\max }\right)$ typically are around $75 \mathrm{ng} / \mathrm{mL}$ or less with a

64 single dose of 500mg of resveratrol (Boocock et al. 2007). This is much lower than the in vitro

65 concentrations shown to have pharmacological benefits $(>1000 \mathrm{ng} / \mathrm{mL})$, which has been

66 achieved in plasma of rodents treated with large doses of resveratrol (de la Lastra and Villegas

67 2005). This suggests that a higher dose, or enhanced bioavailability, is potentially needed to

68 produce beneficial effects in humans. Piperine has been used in combination with

69 neutraceuticals such as coenzyme Q10, beta-carotene, and resveratrol to enhance their

70 bioavailability and bioefficacy (Badmaev et al. 1999; Badmaev et al. 2000; Johnson et al. 2011;

71 Wightman et al. 2014). Therefore, it seems plausible that co-supplementing resveratrol with

72 piperine could lead to enhanced bioavailability and bioefficacy of resveratrol and improve the

73 ability to produce physiological benefits in humans yet minimize the potential risks associated

74 with high doses (Mennen et al. 2005).

75 The purpose of this study was to evaluate the influence of co-ingesting $500 \mathrm{mg}$ of

76 resveratrol with $10 \mathrm{mg}$ of piperine on changes in muscle mitochondrial capacity with

77 submaximal endurance training in healthy subjects compared to a placebo group. It was

78 hypothesized that 4 weeks of supplementation of resveratrol+piperine in combination with

79 submaximal endurance training would produce a greater increase in muscle mitochondrial

80 capacity than exercise training or resveratrol+piperine alone. 


\section{Methods}

\section{Participants}

A total of 16 healthy young adults (9 males, 7 females) volunteered for the study.

85 Participants were excluded if they were currently taking medications other than oral

86 contraceptives or any vitamin supplements. Participants were also excluded from the study if

87 there exercise regimen involved excessive use of the forearm muscle, such as rowing, rock

88 climbing, or Crossfit. Participants were instructed to maintain exercise and dietary habits and

89 abstain from vigorous forearm activity during the study. Participants were instructed to consume

90 their study pills each morning upon waking. All experimental procedures were approved by the

91 Human Subjects Institutional Review Board at the University of Georgia. Verbal and written

92 explanations of the experimental protocol and associated risks were provided to all participants

93 prior to obtaining written informed consent.

Exercise Training Procedures

This was a longitudinal training study in which participants performed 30 minutes of

97 supervised forearm wrist flexor exercises of the non-dominant arm 3 times per week over 4

98 weeks. The wrist flexor muscles were chosen as the muscle of interest because they are

99 relatively detrained, non-weight bearing muscles compared to muscles of the lower limb. The

100 dominant arm was not trained and used as the untrained control arm for each subject.

101 The exercise training regimen used in this study was a submaximal endurance training

102 protocol similar to previous endurance training programs (Ryan et al. 2013a). During baseline

103 testing, participants performed a maximal voluntary isometric contraction (MVIC) to determine

104 the appropriate weight for exercise training and testing. MVIC was determined using a JAMAR 
105 ® handgrip dynamometer (Sammons Preston Rolyan, Bolingbrook, IL). Participants were given

106 the hand dynamometer and instructed to perform three MVIC's on each hand. Participants were

107 instructed to rest 20 seconds between each contraction and the highest value of the three

108 contractions was recorded. Participants trained with dumbbell weights adjusted to $12-15 \%$ of

109 their MVIC and performed the exercise on a flat surface, such as the arm of a chair, with the

110 elbow at 90 degrees of flexion. Training was performed during the morning hours of the day

111 before 11 am so that the time of training coincided with the time resveratrol reaches peak plasma

112 concentrations after ingestion of pills (Boocock et al. 2007). Participants began training with a

113 contraction frequency of 1 contraction every 3.5 seconds ( 514 contractions per session) during

114 week one. Starting with week two the frequency was increased to 1 contraction every 2.5

115 seconds (720 contractions per session) and remained at this frequency for the remaining 2 weeks

116 of the study.

118 Supplementation Procedures

119 Participants were randomly assigned in a double blind fashion to receive one pill

120 containing $500 \mathrm{mg}$ of resveratrol and one pill containing $10 \mathrm{mg}$ of piperine $(\mathrm{n}=8)$; or two

121 identically appearing placebo pills containing flour $(n=8)$. Participants were asked to consume

122 the pills upon waking each morning. Participants were randomized in a double-blind fashion

123 and given pills by a researcher who was not a member of the investigational team and did not

124 participate in either the training or testing of participants. Randomization was done using

125 randomizer.org. The resveratrol supplement contained 99\% Pure Trans Resveratrol

126 (MegaResveratrol, Danbury, USA) in size "0" vegetable capsules and the piperine supplement

127 (BioPerine $\left.{ }^{\circledR}\right)$ was provided by Sabinsa and contained 95\% Piperine prepared in to size "3" 
128 vegetable capsules. All pills were prepared by the lead researcher and coded by a third party

129 researcher who did not participate in either the training or testing of participants. No member of

130 the investigative team was aware of the contents of the capsules until all training and

131 mitochondrial capacity measurements were completed and analyzed.

133 Mitochondrial Capacity Procedures and Measurements

134 Mitochondrial capacity measurements took 45 minutes and were made at weeks $0,2,3$,

135 and 4. Mitochondrial capacity, measured using near infrared spectroscopy (NIRS) (Ryan et al.

136 2012; Ryan et al. 2013a), yields results highly correlated to gold standard measures of

137 mitochondrial function including magnetic resonance spectroscopy (Ryan et al. 2013b) and

138 biochemical analysis of muscle biopsies (Ryan et al. 2014). The NIRS protocol was performed

139 on both the non-dominant (training arm) and dominant arm (non-training arm). The participant

140 was placed supine on a padded table with the test arm extended 90 degrees from the body. The

141 NIRS probe was placed over the superficial wrist flexor muscles (flexor carpi radialis, palmaris

142 longus, and flexor carpi ulnaris) approximately $2-3 \mathrm{~cm}$ distal to the medial epicondyle of the

143 humerus. A blood pressure cuff (Hokanson SC5, Bellevue, WA) was placed above the elbow

144 joint and was attached to a rapid cuff-inflation system (Hokanson E20 cuff inflator) powered by

145 a 30-gallon commercial air compressor (Husky VT6315, Kenosha, WI).

146 NIRS signals were obtained using a continuous wave NIRS device (Oxymon MK III;

147 Artinis Medical Systems, The Netherlands). Adipose tissue thickness (ATT) was measured at

148 the site of the NIRS probe using B-mode ultrasound (LOGIQe; GE HealthCare, USA). The

149 NIRS probe had two source detector separation distances that were set based on the amount of

150 adipose tissue thickness (ATT) on top of the muscle of interest. The transmitter and receiver on 
151 the NIRS probe were set to a distance at least twice the ATT depth in order to assure NIRS

152 penetration depth was adequate to reach the muscle. NIRS data were collected at $10 \mathrm{~Hz}$. NIRS

153 signals that represent oxygenated $\left(\mathrm{O}_{2} \mathrm{Hb}\right)$ and deoxygenated $(\mathrm{HHb})$ hemoglobin/myoglobin were

154 corrected for changes in blood volume as previously described (Ryan et al. 2012).

155 Mitochondrial capacity was measured using a short bout of voluntary exercise to increase

156 metabolic rate, and the rate of recovery of metabolic rate after the exercise was measured

157 (McCully et al. 2009; Ryan et al. 2013a). The testing protocol consisted of a 5 - 10 second bout

158 of voluntary exercise followed by a series of 17-20 short duration arterial occlusions (5-10

159 seconds) (Figure 1A). The exercise/occlusion protocol was performed twice. The rate of

160 recovery of oxygen consumption measured by the difference signal $\left(\mathrm{Hb}_{\text {difference }}=\mathrm{O}_{2} \mathrm{Hb}-\mathrm{HHB}\right)$

161 was calculated as the slope of the change during arterial occlusion using linear regression. The

162 repeated measures were fit to a monoexponential curve and a mitochondrial rate constant (k) was

163 determined for each fit (Figure 1B). Prior to mitochondrial capacity tests, a five minute

164 ischemic calibration was used to normalize NIRS signal, completely deoxygenating the muscle

165 tissue under the probe. The cuff was then released to obtain a peak hyperemic response which

166 was used to indicate $100 \%$ oxygenation. This calibration was also used to monitor depletion of

167 oxygen saturation during the short bout of exercise in the recovery protocol. Oxygen depletion

168 was kept between $30-50 \%$ of the $100 \%$ hyperemic response to ischemia to reduce the likelihood

169 that rate of oxygen utilization during the ischemic cuff periods were limited by oxygen delivery.

171 Statistical Analysis

172 The differences between baseline testing and time points 2, 3 and 4 were first calculated

173 and a two-way repeated measures ANOVA was used to compare the effect of group 
174 (resveratrol+piperine vs. placebo) and time (difference between weeks compared to baseline) for

175 mitochondrial capacity data in the trained and untrained arms. Examination of individual data

176 indicated considerable variability in initial values of mitochondrial capacity among study

177 participants. ANCOVA was therefore used to control for individual variation in baseline

178 mitochondrial capacity that plausibly could influence responsiveness to the intervention.

179 Because of equipment problems, missing data occurred at week 3 for one individual in the

180 placebo trained arm group and a second individual in the placebo untrained arm. These missing

181 values were replaced with the last value carried forward. Fishers LSD was used for the post-hoc

182 pair-wise comparisons. A two-way repeated measures ANOVA was applied to strength

183 measurement data with a within subjects factor of time (pre- and post-) and a between subjects

184 factor of group. All statistical analysis were performed using SPSS 19.0 (IBM $\mathbb{B}_{\mathbb{B}}$, Armonk, NY).

185 Statistical significance was accepted at $p<0.05$ and all data are presented as means \pm SD.

\section{$\underline{\text { Results }}$} adverse events. The physical characteristics of the participants in this study are shown in Table 1. Compliance was monitored via daily text messages and determined using a post-screening

191 questionnaire which was administered after completion of the study (Wei et al. 2011). Based on 192 the questionnaire, there was a $99.1 \%$ adherence to supplementation and no participant missed 193 more than 2 out of 28 days of capsules. All training sessions were completed by all participants $194(12 / 12)$ resulting in $100 \%$ adherence to exercise training.

195 The statistical analysis of the strength measurements revealed that the interaction was not 196 significant in either the trained arms $(p=0.55)$ or the untrained arms $(p=0.43)$. Baseline strength 
197

198

measurements were not different between the resveratrol group and the placebo group, and did not change during the course of the intervention.

Average mitochondrial rate constants between groups are displayed in Figures 2A and

2B. Examination of individual baseline data indicated substantial heterogeneity among subjects within each group (Figure 3A, 3B). When using baseline mitochondrial capacity values as a covariate in the ANCOVA model, the analysis identified a significant group $\times$ time interaction effect in the trained arm $(p=0.002)$. In the resveratrol+piperine group only, pairwise comparisons indicated significant differences in the change in mitochondrial rate constants from baseline to post testing compared to changes at weeks 2 ( $\Delta \mathrm{k}=0.03$ vs $\Delta \mathrm{k}=0.58, p=0.001$ ) and weeks $3(\Delta \mathrm{k}=0.13$ vs $\Delta \mathrm{k}=0.58, p=0.005)$ (Figure 4). There were no significant differences among time points in the placebo group. When comparing the resveratrol+piperine trained arm to the placebo trained arm, the change from baseline to post testing indicated significant differences between the two groups $(p=0.02)$, the resveratrol+piperine group increasing about $40 \%$ from baseline $(\Delta \mathrm{k}=0.58)$, where the placebo group increased about $10 \%$ from baseline $(\Delta \mathrm{k}=0.13)$. A two-way ANCOVA did not identify a significant interaction effect in the untrained arms between the 2 variables (group $\times$ time) $(p=0.66)$, meaning that the untrained arms NIRS rate constants did not differ significantly between groups or over time.

\section{$\underline{\text { Discussion }}$}

The primary finding in this study was that 4 weeks of resveratrol and piperine supplementation combined with a submaximal endurance training stimulus significantly increased mitochondrial oxidative capacity, compared to exercise training with a placebo. Previous studies have shown administration of resveratrol enhances AMPK and SIRT1, leading 
220 to activation of PGC-1 $\alpha$ (Lagouge et al. 2006; Timmers et al. 2011; Ungvari et al. 2011; Menzies

221 et al. 2013) which is the primary pathway associated with endurance exercise adaptations leading

222 to mitochondrial biogenesis (Winder et al. 2000; Jager et al. 2007; Hood 2009). Therefore,

223 resveratrol along with exercise training, could enhance this pathway to a greater extent than

224 exercise alone. This is supported by studies in mice and rats that have shown resveratrol

225 enhances physiological adaptations to exercise training (Dolinsky et al. 2012; Hart et al. 2013;

226 Menzies et al. 2013), which are consistent with the findings in this study demonstrating that

227 mitochondrial capacity was increased after 4-weeks of arm training in the resveratrol+piperine

228 group, but not the placebo group.

229 Recent studies investigating the interactive effects of training and resveratrol

230 supplementation in humans have produced mixed results. Studies performed in obese and

231 diabetic populations have been consistent with rodent models, indicating increases in skeletal

232 muscle SIRT1 and AMPK protein levels with resveratrol supplementation (Timmers et al. 2011;

233 Goh et al. 2014). However, studies combining exercise and resveratrol supplementation in

234 healthy adults have reported attenuated training induced adaptations with supplementation of

235 resveratrol. A study examining the effects of 4 weeks of high-intensity interval training (HIIT)

236 of the legs found that mitochondrial biogenesis-related gene expression in skeletal muscle was

237 unchanged from baseline with resveratrol supplementation. Specifically, PGC-1 $\alpha$ and SIRT1

238 gene expression were increased in the placebo group, and unchanged in the resveratrol group

239 after the training period, indicating resveratrol supplementation may attenuate mitochondrial

240 adaptations to leg exercise training (Scribbans et al. 2014). Another study examining the effects

241 of 8 weeks of a daily dosage of $250 \mathrm{mg}$ of resveratrol in combination with high intensity exercise

242 training also found no effects of resveratrol on SIRT1, AMPK, and PGC-1 $\alpha$. However, exercise 
243 training alone did increase these mitochondrial markers (Olesen et al. 2014). Other studies have

244 reported resveratrol has no effect on physiological adaptations to training, such as whole body

245 maximal oxygen uptake (Gliemann et al. 2013; Voduc et al. 2014; Macedo et al. 2015). A

246 potential difference between these studies and the current study may be due to the muscle group

247 trained, the intensity of the exercise training regimens or the dosage amounts. For example,

248 Scribbans et al. (Scribbans et al. 2014), Gliemann et al. (Gliemann et al. 2013) and Olesen et al.

249 (Olesen et al. 2014) performed leg HIIT protocols 3 times per week. Based on our data, it seems

250 possible that resveratrol+piperine could enhance adaptations to lower intensity training stimuli in

251 relatively untrained muscle, controlling for baseline differences in mitochondrial capacity.

252 Future studies are needed to systematically examine whether resveratrol differentially modifies

253 adaptations to low vs. high intensity exercise training, or arm vs. leg training.

254 An additional consideration is the amount of resveratrol necessary to produce

255 physiological changes. Scribbans et al. (Scribbans et al. 2014), Gliemann et al. (Gliemann et al.

256 2013), and Oleson et al. (Olesen et al. 2014) supplemented with 250mg/day or less of resveratrol.

257 Increasing the dosage of resveratrol given in this study to $500 \mathrm{mg}$ instead of the $250 \mathrm{mg}$ given in

258 the previous studies may have been important in inducing beneficial adaptations and increasing

259 mitochondrial capacity. Significant enhancement of mitochondrial capacity did not occur until

260 the last measurement in the resveratrol group. This suggests that supplementation of resveratrol

261 may need a preloading period to significantly increase blood concentrations to produce

262 physiological effects (Almeida et al. 2009; Brown et al. 2010). Another concern is the

263 bioavailability and bioefficacy of resveratrol. In this study, resveratrol was combined with

264 piperine in hopes to enhance the bioavailability and bioefficacy of resveratrol. Resveratrol is

265 rapidly metabolized and results in the formation of various resveratrol metabolites and 
266 conjugates. These conjugates could act differently than the parent compound, possibly inducing

267 different physiological effects, however further research needs to be done to better understand

268 the biological activity of resveratrol and its conjugates (Baur and Sinclair 2006; Brown et al.

269 2010). The use of piperine in the current study could have been a key component due to its

270 reported ability to inhibit glucuronidation of polyphenol antioxidants (Shoba et al. 1998;

271 Lambert et al. 2004; Srinivasan 2007), therefore possibly preventing extensive metabolism of

272 resveratrol and the formation of resveratrol conjugates, allowing a greater amount of

273 untransformed resveratrol to be absorbed. Combining resveratrol with piperine has been shown

274 to increase the absorption and maximum serum levels of resveratrol concentration by $\sim 1500 \%$ in

275 mice given $100 \mathrm{mg} / \mathrm{kg}$ resveratrol and $10 \mathrm{mg} / \mathrm{kg}$ piperine (Johnson et al. 2011). Along these

276 lines, it has also been reported that the bioefficacy of resveratrol is also enhanced when co-

277 supplementing with piperine in healthy human subjects. This study also confirmed that

278 resveratrol metabolites and conjugate serum levels were lower with the addition of piperine,

279 indicating piperine's inhibition of resveratrol glucuronidation (Wightman et al. 2014). Therefore,

280 combining piperine with resveratrol could have been a contributing factor to the changes

281 observed in this study.

282 This study is also different than previous studies in our use of a low intensity exercise

283 stimulus instead of a high-intensity exercise stimulus combined with resveratrol

284 supplementation. The low intensity stimulus was chosen because previous literature has only

285 assessed resveratrol supplementation in combination with high intensity exercise training and

286 was chosen to mimic an intensity that is feasible for all populations, including patient

287 populations who may be unable to exercise at high intensities. It is interesting that the

288 submaximal exercise stimulus in this study was not sufficient to increase mitochondrial capacity 
within the placebo group, only 3 of 8 individuals within the placebo group exhibited training-

290 induced increases in mitochondrial rate constants from baseline. Conversely, in the

291 resveratrol+piperine group, supplementation and training increased mitochondrial rate constants

292 in 7 of 8 participants. Moreover, no changes in mitochondrial capacity were observed in the

293 untrained arm of the resveratrol+piperine group. Taken together, these findings indicate that

294 combination of exercise and resveratrol is needed for eliciting muscle mitochondrial adaptations

295 to low-intensity training programs such as that used in the present study. We speculate that

296 small changes at the molecular level with this submaximal stimulus could have occurred, but the

297 signals were not enough to elicit training adaptations at the physiological level in the placebo

298 trained arm. This conclusion is supported by Timmers et al. who detected resveratrol-induced

299 increases in muscle AMPK, PGC-1 $\alpha$, and citrate synthase expression- all biomarkers of

300 mitochondrial biogenesis- but did not find a change in mitochondrial function in vivo when

301 measuring phosphocreatine recovery rate after acute exercise (Timmers et al. 2011).

302 Some of the limitations of this study were that plasma levels of resveratrol in the blood

303 were not assessed, muscle tissue samples were not taken to determine activation of mitochondrial

304 biogenesis- related signaling pathways, and the effect of piperine on mitochondrial capacity was

305 not assessed independent of co-supplementation with resveratrol. Measuring resveratrol

306 concentrations in the blood could have led to a better understanding of the bioavailability and

307 dosage amounts needed to induce physiological effects, and how repeated dosing at the amount

308 given over 28 days effects $\mathrm{C}_{\max }$ values. Measuring circulating resveratrol could have provided

309 insight as to whether piperine did indeed raise resveratrol levels in the blood, and would have

310 served as another indicator of participant compliance. Diet was also not controlled or accounted

311 for in this study, which may be a confounding variable. However, dietary intake of resveratrol is 
312 estimated to be small relative to the doses administered in this study. Muscle tissue samples

313 could have provided more insight in to the effects of training and resveratrol+piperine on

314 mitochondrial capacity, as well as the mechanistic pathways resveratrol activates. Muscle biopsy

315 analysis would have also made this study easier to compare to others indicating activation of

316 proteins such as SIRT1 and AMPK. Finally, future studies may determine the independent and

317 combined effects of piperine and resveratrol on mitochondrial adaptations to training (Srinivasan 318 2007).

\section{$\underline{\text { Conclusions }}$}

321 This is the first study to show that the combination of resveratrol+piperine

322 supplementation and low-intensity, submaximal exercise training enhances mitochondrial

323 capacity in humans. Using piperine as a bioenhancer may be a key component when

324 supplementing with resveratrol, although further studies are needed to determine whether

325 piperine definitively augments resveratrol-mediated improvements in mitochondrial training

326 adaptations. More studies are also needed to determine the cellular mechanisms by which

327 resveratrol increases mitochondrial capacity and the optimal dosage of resveratrol required to 328 safely elicit these physiological adaptations.

\section{$\underline{\text { Acknowledgements }}$}

331 We would like to thank Sabinsa (C) for providing the piperine for this study. We would 332 also like to thank Melissa Erickson, MS, for managing the blinding of this study.

333 The authors' contributions were as follows- KP, NJ, PO, KM: actively involved in the 334 planning of the research and in writing the manuscript; KP: conducted research, collected and 
335 analyzed data. All authors contributed to and reviewed the final publication. None of the 336 authors had any conflicts of interest. 


\section{$\underline{\text { References }}$}

Almeida, L., Vaz-da-Silva, M., Falcao, A., Soares, E., Costa, R., Loureiro, A.I., FernandesLopes, C., Rocha, J.F., Nunes, T., Wright, L., andSoares-da-Silva, P. 2009. Pharmacokinetic and safety profile of trans-resveratrol in a rising multiple-dose study in healthy volunteers. Mol Nutr Food Res 53 Suppl 1: S7-15.

Amri, A., Chaumeil, J.C., Sfar, S., andCharrueau, C. 2012. Administration of resveratrol: What formulation solutions to bioavailability limitations? J Control Release 158: 182-93.

Badmaev, V., Majeed, M., andNorkus, E.P. 1999. Piperine, an alkaloid derived from black pepper increases serum response of beta-carotene during 14-days of oral beta-carotene supplementation. Nutrition Research 19: 381-388.

Badmaev, V., Majeed, M., andPrakash, L. 2000. Piperine derived from black pepper increases the plasma levels of coenzyme Q10 following oral supplementation. J Nutr Biochem 11: 109-13.

Baur, J.A. and Sinclair, D.A. 2006. Therapeutic potential of resveratrol: the in vivo evidence. Nat Rev Drug Discov 5: 493-506.

Boocock, D.J., Faust, G.E., Patel, K.R., Schinas, A.M., Brown, V.A., Ducharme, M.P., Booth, T.D., Crowell, J.A., Perloff, M., Gescher, A.J., Steward, W.P., andBrenner, D.E. 2007. Phase I dose escalation pharmacokinetic study in healthy volunteers of resveratrol, a potential cancer chemopreventive agent. Cancer Epidemiol Biomarkers Prev 16: 1246-52.

Brown, V.A., Patel, K.R., Viskaduraki, M., Crowell, J.A., Perloff, M., Booth, T.D., Vasilinin, G., Sen, A., Schinas, A.M., Piccirilli, G., Brown, K., Steward, W.P., Gescher, A.J., andBrenner, D.E. 2010. Repeat dose study of the cancer chemopreventive agent resveratrol in healthy volunteers: safety, pharmacokinetics, and effect on the insulin-like growth factor axis. Cancer Res 70: 9003-11.

de la Lastra, C.A. and Villegas, I. 2005. Resveratrol as an anti-inflammatory and anti-aging agent: mechanisms and clinical implications. Mol Nutr Food Res 49: 405-30.

Dolinsky, V.W., Jones, K.E., Sidhu, R.S., Haykowsky, M., Czubryt, M.P., Gordon, T., andDyck, J.R. 2012. Improvements in skeletal muscle strength and cardiac function induced by resveratrol during exercise training contribute to enhanced exercise performance in rats. $\mathbf{J}$ Physiol 590: 2783-99.

Gliemann, L., Schmidt, J.F., Olesen, J., Bienso, R.S., Peronard, S.L., Grandjean, S.U., Mortensen, S.P., Nyberg, M., Bangsbo, J., Pilegaard, H., andHellsten, Y. 2013. Resveratrol blunts the positive effects of exercise training on cardiovascular health in aged men. $\mathbf{J}$ Physiol 591: 5047-59.

Goh, K.P., Lee, H.Y., Lau, D.P., Supaat, W., Chan, Y.H., andKoh, A.F. 2014. Effects of resveratrol in patients with type 2 diabetes mellitus on skeletal muscle SIRT1 expression and energy expenditure. Int J Sport Nutr Exerc Metab 24: 2-13.

Hardy, G. 2000. Nutraceuticals and functional foods: introduction and meaning. Nutrition 16: 688-9.

Hart, N., Sarga, L., Csende, Z., Koltai, E., Koch, L.G., Britton, S.L., Davies, K.J., Kouretas, D., Wessner, B., andRadak, Z. 2013. Resveratrol enhances exercise training responses in rats selectively bred for high running performance. Food Chem Toxicol 61: 53-9.

Hood, D.A. 2009. Mechanisms of exercise-induced mitochondrial biogenesis in skeletal muscle. Appl Physiol Nutr Metab 34: 465-72. 
Jager, S., Handschin, C., St-Pierre, J., andSpiegelman, B.M. 2007. AMP-activated protein kinase (AMPK) action in skeletal muscle via direct phosphorylation of PGC-1alpha. Proc Natl Acad Sci U S A 104: 12017-22.

Johnson, J.J., Nihal, M., Siddiqui, I.A., Scarlett, C.O., Bailey, H.H., Mukhtar, H., andAhmad, N. 2011. Enhancing the bioavailability of resveratrol by combining it with piperine. Mol Nutr Food Res 55: 1169-76.

Kulkarni, S.S. and Canto, C. 2014. The molecular targets of resveratrol. Biochim Biophys Acta. Lagouge, M., Argmann, C., Gerhart-Hines, Z., Meziane, H., Lerin, C., Daussin, F., Messadeq, N., Milne, J., Lambert, P., Elliott, P., Geny, B., Laakso, M., Puigserver, P., andAuwerx, J. 2006. Resveratrol improves mitochondrial function and protects against metabolic disease by activating SIRT1 and PGC-1alpha. Cell 127: 1109-22.

Lambert, J.D., Hong, J., Kim, D.H., Mishin, V.M., andYang, C.S. 2004. Piperine enhances the bioavailability of the tea polyphenol (-)-epigallocatechin-3-gallate in mice. J Nutr 134: 1948-52.

Macedo, R.C., Vieira, A., Marin, D.P., andOtton, R. 2015. Effects of chronic resveratrol supplementation in military firefighters undergo a physical fitness test - A placebocontrolled, double blind study. Chem Biol Interact 227c: 89-95.

McCully, K.K., Turner, T.N., Langley, J., andZhao, Q. 2009. The reproducibility of measurements of intramuscular magnesium concentrations and muscle oxidative capacity using 31P MRS. Dyn Med 8: 5.

Mennen, L.I., Walker, R., Bennetau-Pelissero, C., andScalbert, A. 2005. Risks and safety of polyphenol consumption. Am J Clin Nutr 81: 326s-329s.

Menzies, K.J., Singh, K., Saleem, A., andHood, D.A. 2013. Sirtuin 1-mediated effects of exercise and resveratrol on mitochondrial biogenesis. J Biol Chem 288: 6968-79.

Murase, T., Haramizu, S., Ota, N., andHase, T. 2009. Suppression of the aging-associated decline in physical performance by a combination of resveratrol intake and habitual exercise in senescence-accelerated mice. Biogerontology 10: 423-34.

Olesen, J., Gliemann, L., Bienso, R., Schmidt, J., Hellsten, Y., andPilegaard, H. 2014. Exercise training, but not resveratrol, improves metabolic and inflammatory status in skeletal muscle of aged men. J Physiol 592: 1873-86.

Ryan, T.E., Brophy, P., Lin, C.T., Hickner, R.C., andNeufer, P.D. 2014. Assessment of in vivo skeletal muscle mitochondrial respiratory capacity in humans by near-infrared spectroscopy: a comparison with in situ measurements. J Physiol 592: 3231-41.

Ryan, T.E., Erickson, M.L., Brizendine, J.T., Young, H.J., andMcCully, K.K. 2012. Noninvasive evaluation of skeletal muscle mitochondrial capacity with near-infrared spectroscopy: correcting for blood volume changes. J Appl Physiol (1985) 113: 175-83.

Ryan, T.E., Southern, W.M., Brizendine, J.T., andMcCully, K.K. 2013a. Activity-induced changes in skeletal muscle metabolism measured with optical spectroscopy. Med Sci Sports Exerc 45: 2346-52.

Ryan, T.E., Southern, W.M., Reynolds, M.A., andMcCully, K.K. 2013b. A cross-validation of near-infrared spectroscopy measurements of skeletal muscle oxidative capacity with phosphorus magnetic resonance spectroscopy. J Appl Physiol (1985) 115: 1757-66.

Scalbert, A., Johnson, I.T., andSaltmarsh, M. 2005. Polyphenols: antioxidants and beyond. Am J Clin Nutr 81: 215s-217s. 
Schrauwen, P. and Timmers, S. 2014. Can resveratrol help to maintain metabolic health? Proc Nutr Soc 73: 271-7.

Scribbans, T.D., Ma, J.K., Edgett, B.A., Vorobej, K.A., Mitchell, A.S., Zelt, J.G., Simpson, C.A., Quadrilatero, J., andGurd, B.J. 2014. Resveratrol supplementation does not augment performance adaptations or fibre-type-specific responses to high-intensity interval training in humans. Appl Physiol Nutr Metab 39: 1305-13.

Shoba, G., Joy, D., Joseph, T., Majeed, M., Rajendran, R., andSrinivas, P.S. 1998. Influence of piperine on the pharmacokinetics of curcumin in animals and human volunteers. Planta Med 64: 353-6.

Smoliga, J.M., Baur, J.A., andHausenblas, H.A. 2011. Resveratrol and health--a comprehensive review of human clinical trials. Mol Nutr Food Res 55: 1129-41.

Srinivasan, K. 2007. Black pepper and its pungent principle-piperine: a review of diverse physiological effects. Crit Rev Food Sci Nutr 47: 735-48.

Timmers, S., Konings, E., Bilet, L., Houtkooper, R.H., van de Weijer, T., Goossens, G.H., Hoeks, J., van der Krieken, S., Ryu, D., Kersten, S., Moonen-Kornips, E., Hesselink, M.K., Kunz, I., Schrauwen-Hinderling, V.B., Blaak, E.E., Auwerx, J., andSchrauwen, P. 2011. Calorie restriction-like effects of 30 days of resveratrol supplementation on energy metabolism and metabolic profile in obese humans. Cell Metab 14: 612-22.

Ungvari, Z., Sonntag, W.E., de Cabo, R., Baur, J.A., andCsiszar, A. 2011. Mitochondrial protection by resveratrol. Exerc Sport Sci Rev 39: 128-32.

Voduc, N., la Porte, C., Tessier, C., Mallick, R., andCameron, D.W. 2014. Effect of resveratrol on exercise capacity: a randomized placebo-controlled crossover pilot study. Appl Physiol Nutr Metab 39: 1183-7.

Wei, J., Hollin, I., andKachnowski, S. 2011. A review of the use of mobile phone text messaging in clinical and healthy behaviour interventions. J Telemed Telecare 17: 41-8.

Wightman, E.L., Reay, J.L., Haskell, C.F., Williamson, G., Dew, T.P., andKennedy, D.O. 2014. Effects of resveratrol alone or in combination with piperine on cerebral blood flow parameters and cognitive performance in human subjects: a randomised, double-blind, placebo-controlled, cross-over investigation. Br J Nutr 112: 203-13.

Winder, W.W., Holmes, B.F., Rubink, D.S., Jensen, E.B., Chen, M., andHolloszy, J.O. 2000. Activation of AMP-activated protein kinase increases mitochondrial enzymes in skeletal muscle. J Appl Physiol (1985) 88: 2219-26. 
Table 1. Participant characteristics $(\mathrm{N}=16)$

\begin{tabular}{lllll}
\hline & \multicolumn{2}{c}{ Placebo $(\mathrm{n}=8)$} & \multicolumn{2}{c}{ Resveratrol $(\mathrm{n}=8)$} \\
\cline { 2 - 5 } & Males $(\mathrm{n}=5)$ & Females $(\mathrm{n}=3)$ & Males $(\mathrm{n}=4)$ & Females $(\mathrm{n}=4)$ \\
\hline Age $(\mathrm{y})$ & $21.0 \pm 2.4$ & $19.7 \pm 0.6$ & $20.0 \pm 0.8$ & $19.0 \pm 0.8$ \\
Height $(\mathrm{cm})$ & $176.9 \pm 5.1$ & $166.0 \pm 16.5$ & $175.9 \pm 7.0$ & $162.9 \pm 7.9$ \\
Body mass $(\mathrm{kg})$ & $75.4 \pm 13.1$ & $58.1 \pm 18.0$ & $78.3 \pm 17.0$ & $59.1 \pm 3.5$ \\
ATT $(\mathrm{mm})$ & & & & \\
Nondominant Arm & $3.6 \pm 1.1$ & $5.5 \pm 1.4$ & $4.8 \pm 1.6$ & $6.1 \pm 1.8$ \\
Dominant Arm & $3.4 \pm 1.1$ & $6.0 \pm 2.4$ & $4.4 \pm 1.4$ & $5.7 \pm 1.1$ \\
\hline
\end{tabular}

Note: Data are presented as means $\pm S D$. All measures were made at the start of the study. ATT, adipose tissue thickness. 


\section{$\underline{\text { Figure Legends }}$}

Figure 1: (A) NIRS oxygenated hemoglobin/myoglobin signal during a mitochondrial capacity recovery protocol, consisting of $\sim 5-10$ seconds of voluntary exercise followed by a series of short duration arterial occlusions. (B) Results from the NIRS recovery test measuring the rate of recovery of metabolic rate after $\sim 5-10$ seconds of voluntary exercise.

Figure 2: (A) Mitochondrial rate constant (k) for the recovery of muscle oxidative capacity in the resveratrol (closed diamonds) and placebo (open diamonds) trained arms. (B) Mitochondrial rate constant $(\mathrm{k})$ for the recovery of muscle oxidative capacity in the resveratrol (closed circles) and placebo (open circles) untrained arms.

Figure 3: (A) Individual subjects pre- and post-intervention mitochondrial rate constants (k) for the recovery of muscle oxidative capacity in the placebo trained arm. (B) Individual subjects preand post-intervention mitochondrial rate constants $(\mathrm{k})$ for the recovery of muscle oxidative capacity in the resveratrol training arm.

Figure 4: ANCOVA change scores of mitochondrial rate constants from baseline at weeks 2, 3, and 4 in the placebo (open boxes) and resveratrol (dark boxes) trained arms. * Significantly different from changes at weeks 2 and $3(p<0.05)$. $\uparrow$ Significantly different from placebo change at week $4(p<0.05)$. 


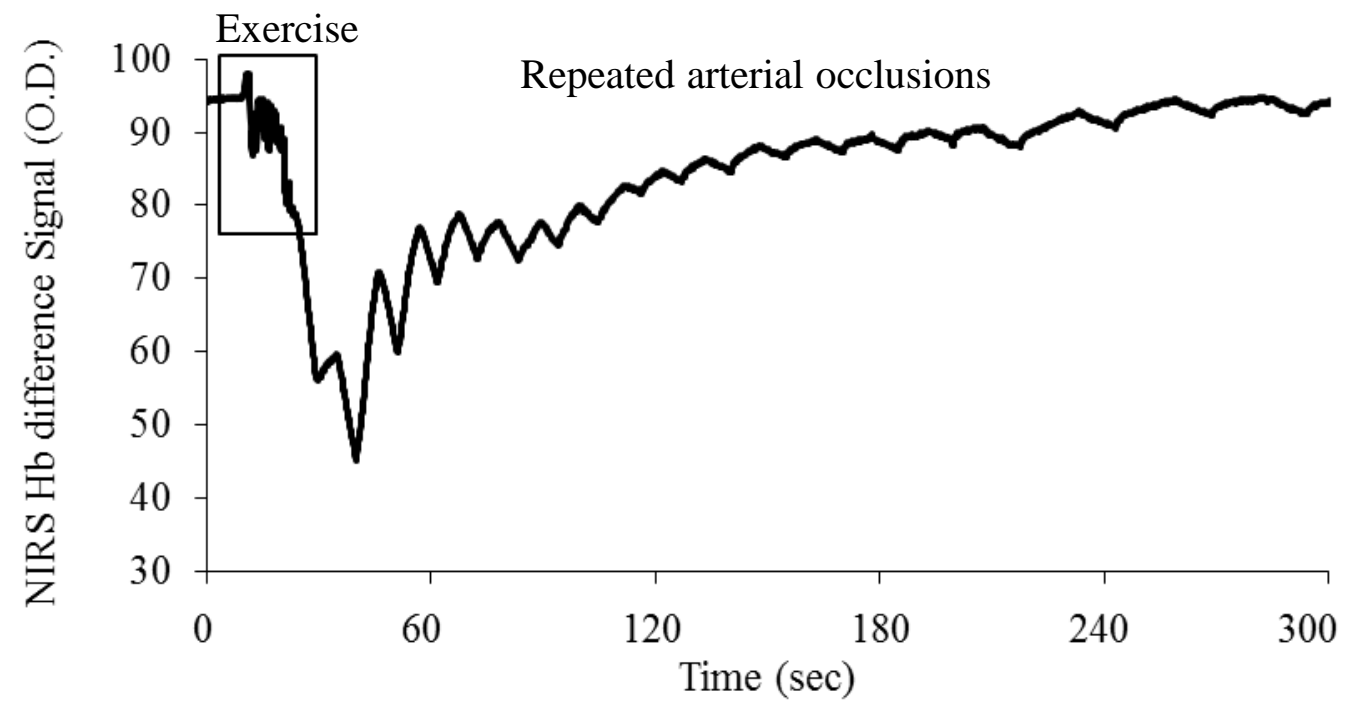

B

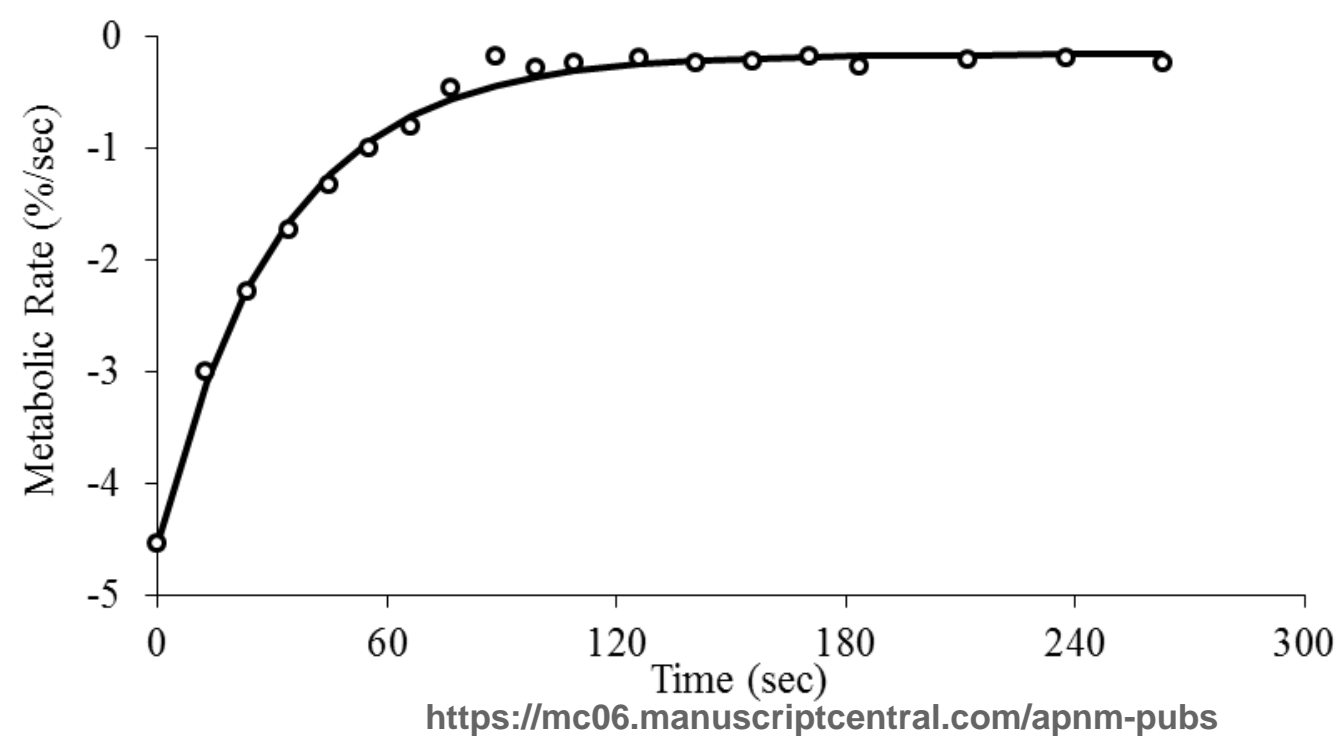




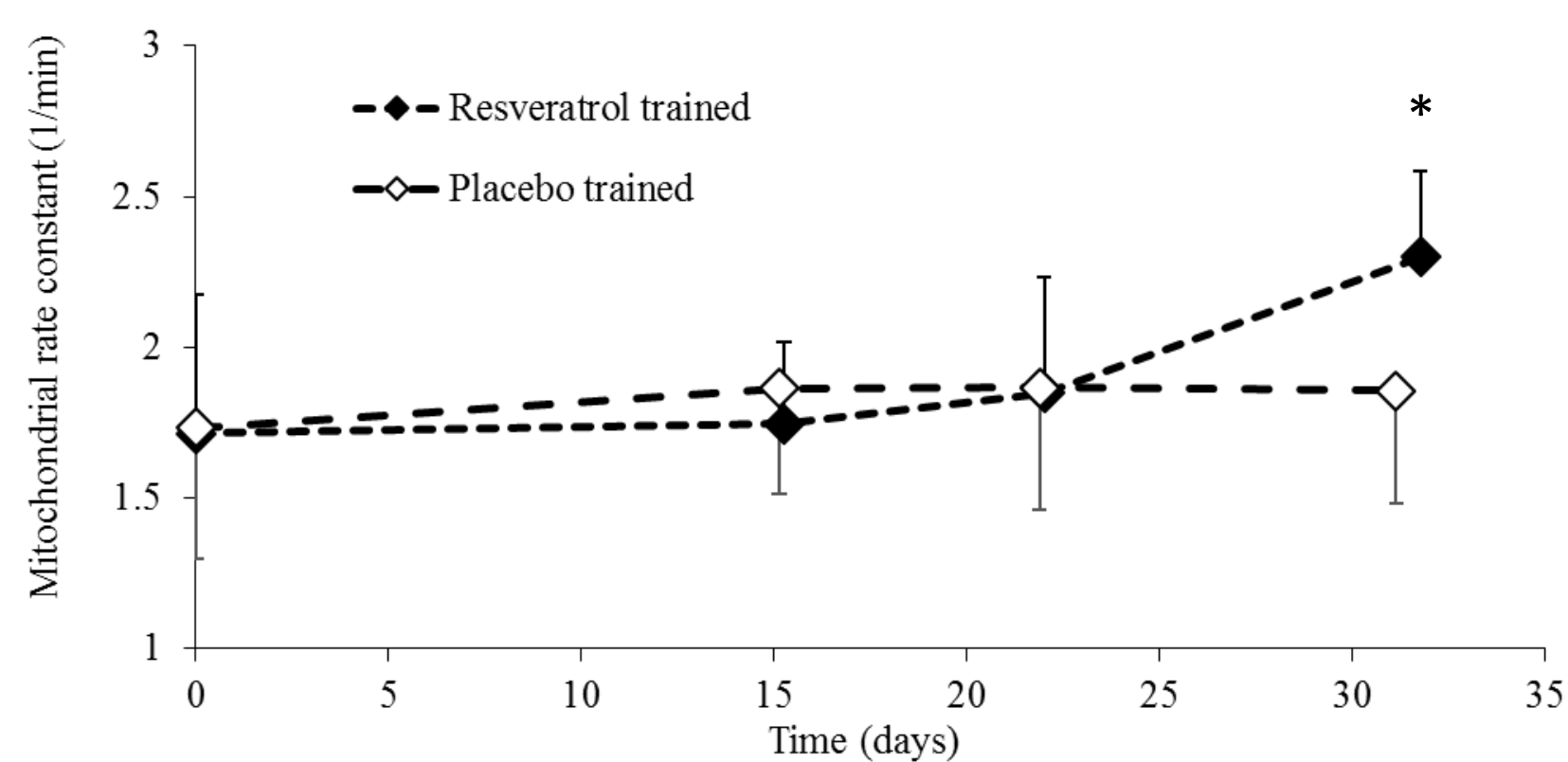

B

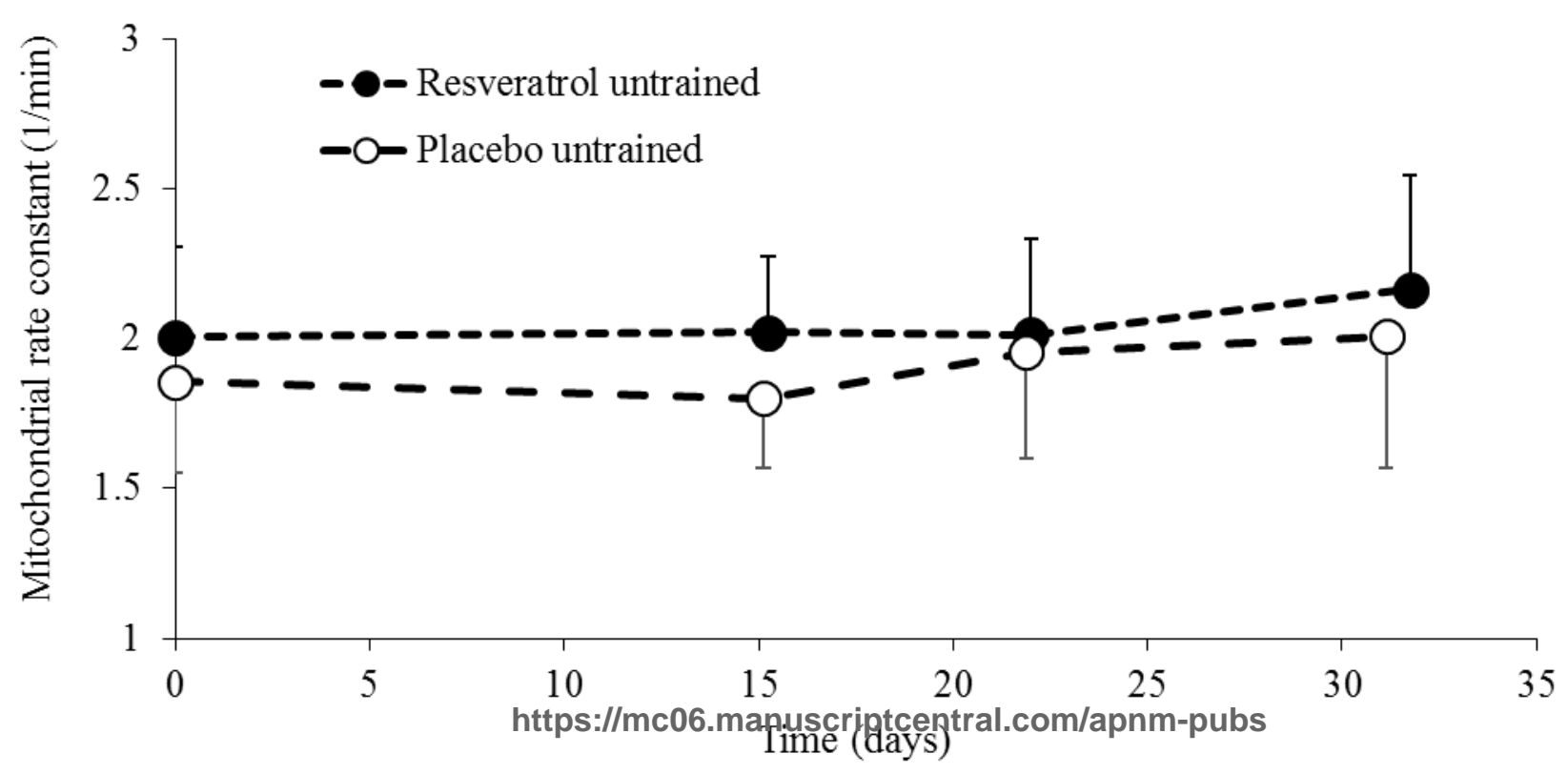


A

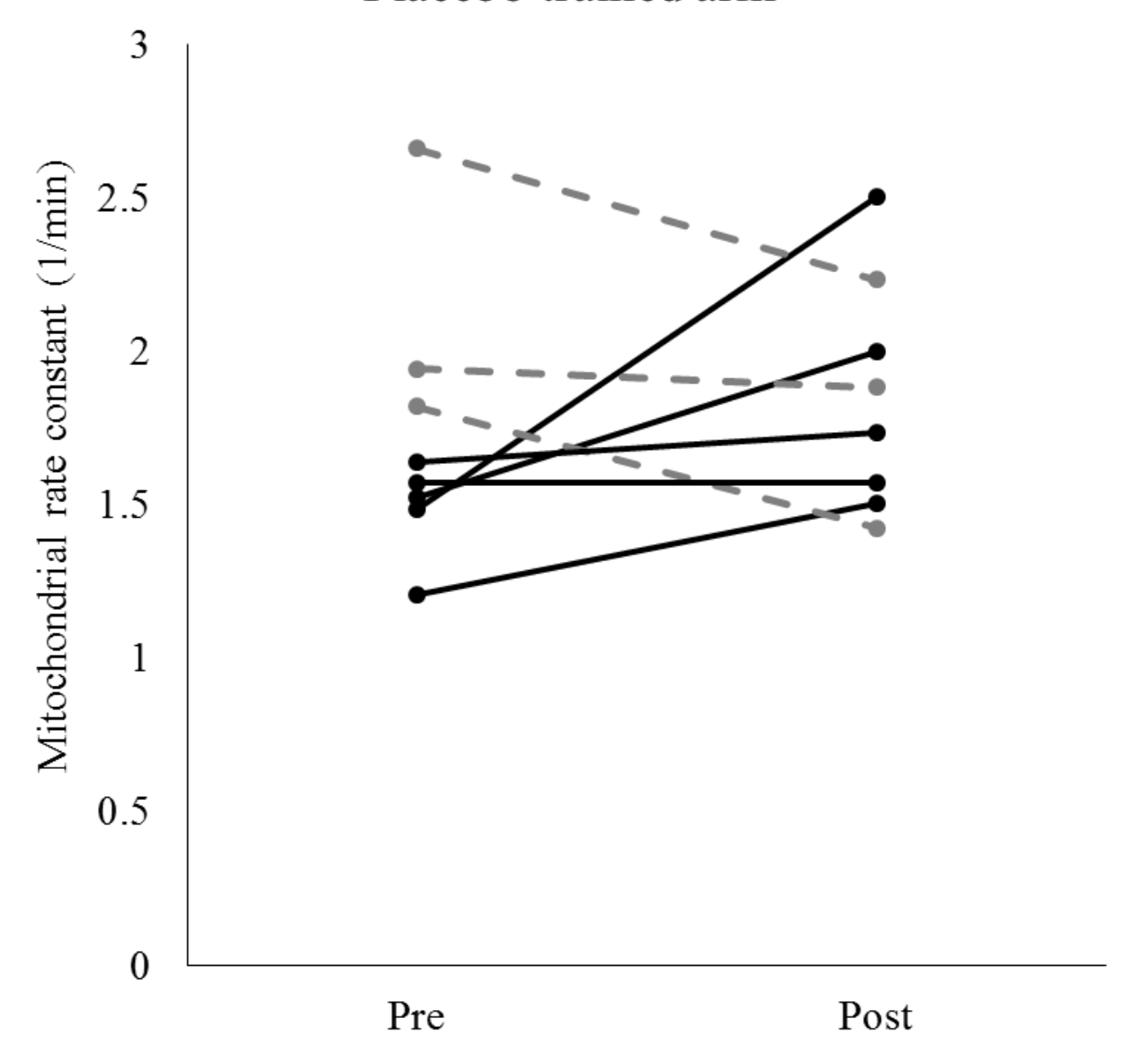

B

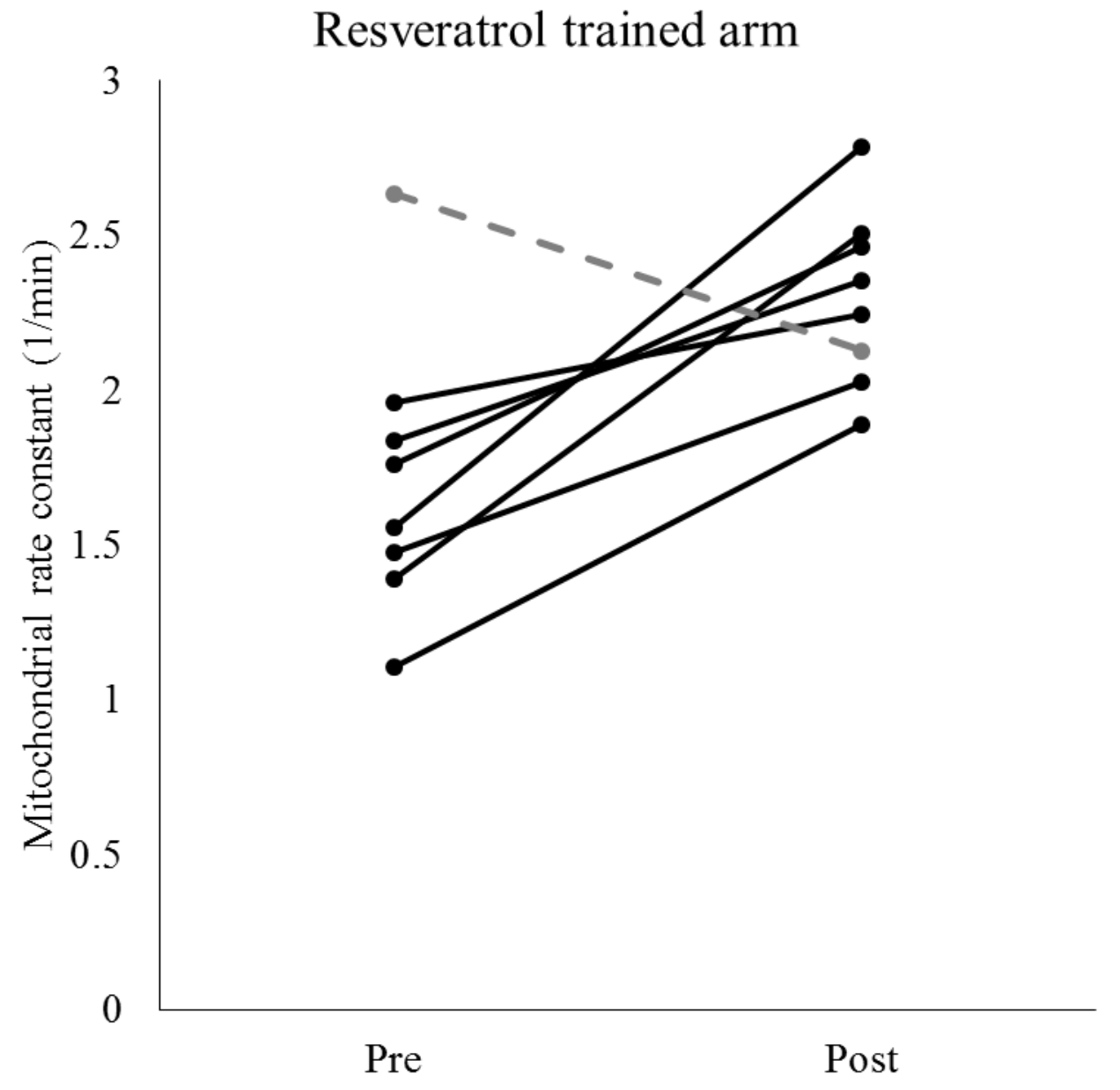




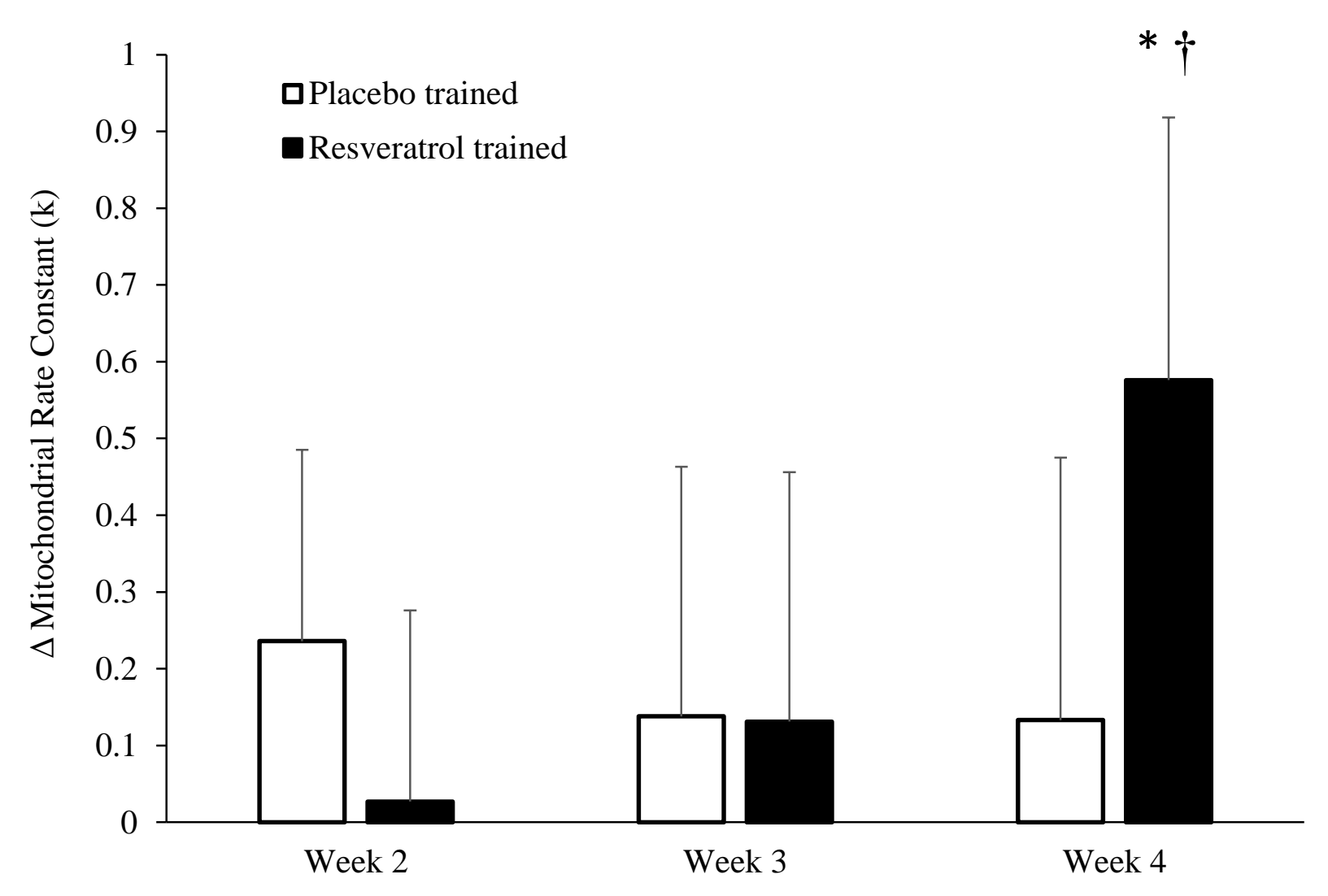

https://mc06.manuscriptcentral.com/apnm-pubs 